\title{
Joint Subchannel Allocation and Hybrid Precoding Design for mmWave Multi-user OFDMA Systems
}

\author{
Vu Nguyen $\mathrm{Ha}^{\dagger}$, Duy H. N. Nguyen ${ }^{\ddagger}$, and Jean-Francois Frigon ${ }^{\dagger}$ \\ ${ }^{\dagger}$ Poly-Grames Research Center, École Polytechnique de Montréal, Montreal, Quebec, Canada \\ ${ }_{\ddagger}^{\ddagger}$ Department of Electrical and Computer Engineering, San Diego State University, CA, USA \\ vu.ha-nguyen@polymtl.ca, duy.nguyen@sdsu.edu, and j-f.frigon@polymtl.ca
}

\begin{abstract}
This paper studies hybrid precoding (HP) for mmWave multi-user OFDMA systems with sub-carrier allocation (SA) consideration. Constrained by a computation limit on the total number of data streams that can be processed, we aim to jointly optimize the SA and HP design to maximize the system sum-rate. This optimization is first formulated as a computation sparsity-constrained HP design problem, which is non-convex and challenging to solve. We then propose two-stage solution approach to tackle the problem. In stage one, we optimize the fully digital precoding (FDP) considering the computation sparsity constraint. In the second stage, we exploit an alternating MMSE minimization algorithm to reconstruct the HP's based on the achieved FDP. A novel analog precoding design, namely "Projected-Gradient-Descent based", is then proposed to optimize the analog part of the HP's.
\end{abstract}

\section{INTRODUCTION}

The demand for high capacity in the future wireless cellular networks drives the search for new radio spectrum resources in the millimeter wave (mmWave) spectrum (between $30 \mathrm{GHz}$ and $300 \mathrm{GHz}$ ) [1]. Thanks to the band's short wavelength, a large number of antenna elements in a sizable space can be employed for mmWave systems. Hence, multiple data streams can be transmitted for multiple users via the spatial multiplexing which results in significant improvement in spectral efficiency [2]. However, a large number of antennas will require the prohibitively high cost and power consumption for multi-user precoding implementation. Therefore, the tranditional MIMO transceiver architecture is not suitable for the mmWave hardware technologies [2].

Recently, HP has been considered as a practical alternative for mmWave systems. This proposed transceiver architecture reduces the number of RF chains by accomplishing a digital precoder and an analog precoder [2], [3]. While research on HP for mmWave system is plentiful [3], a few existing papers have studied resource allocation problems for MU-OFDMA HB systems. The work in [4] has proposed a HP architecture for singlestream MIMO-OFDM to maximize either the signal strength or the sum-rate (SR) over different sub-carriers 978-1-5386-3531-5/17/\$31.00 (c) 2017 IEEE
(SCs). [5] considered the time-slot allocation for the time division multiple access mmWave WPANs and [6] investigated the user scheduling problem for downlink multi-user HP massive MIMO systems.

To the best of our knowledge, downlink MU-OFDMA HB design with considerations on the limited total number of transmitted data streams has not been studied in existing literature. This paper aims to fill this gap where the joint SA and HP design for mmWave MU-OFDMA system is studied to maximize the system SR under a constraint on the number of data streams. Unfortunately, the constraint presents a major obstacle in solving the optimization since it is composed of integer variables. In this paper, we present two-stage based solution approach to this difficult non-convex mixed-integer optimization problem. This solution is based on the premise that near-optimal HP can be designed by approximating an optimal fully digital design [2]. To deal with the sparsity constraint, we develop a general $\ell 1$-norm reweighted solution by regularizing the sparsity function into an approximated linear form and iteratively solving the approximated problem. In addition, an alternative method is employed for designing HP in stage two. For performance evaluation purpose, we also present a joint SA and HP algorithm as referencing benchmark.

\section{SySTEM MODEL}

Consider the downlink mmWave MU-OFDMA HB system as illustrated in Fig. 1 where a base station (BS) equipped with $N_{\mathrm{T}}$ antennas and $N_{\mathrm{RF}}$ RF chains serves $K$ single-antenna users over $S$ SCs. Let $\mathcal{K}$ and $\mathcal{S}$ be the sets of all users and SCs. If SC $s$ is assigned to user $k$, a digital precoding (DP) vector $\mathbf{w}_{k, s} \in \mathbb{C}^{N_{\mathrm{RF}}}$ is applied to the data symbol $x_{k, s} \in \mathbb{C}$, intended for user $k$ at this very $\mathrm{SC}\left(\left|x_{k, s}\right|=1\right)$. Denote $a_{k, s}$ as a binary indicator where $a_{k, s}=1$ or 0 if user $k$ is assigned SC $s$ or not.

Utilizing the HP, the BS first applies all DP vector $\mathbf{w}_{k, s}$ 's to the corresponding symbol sequences $x_{k, s}$ 's before employing an $N_{\mathrm{T}} \times N_{\mathrm{RF}}$ AP matrix $\mathrm{A}$ to map the RF signals from the $N_{\mathrm{RF}} \mathrm{RF}$ chains to the $N_{\mathrm{T}}$ antennas. In this work, we consider the fully-connected RF chains 


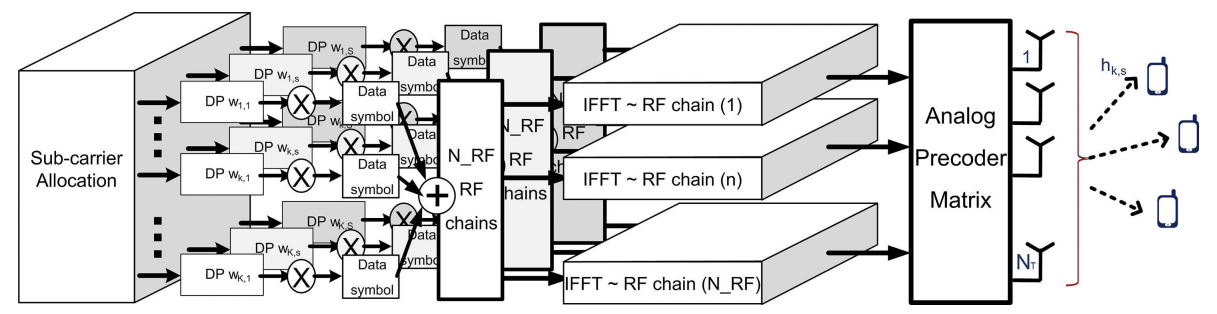

Fig. 1. Diagram of a mmWave OFDMA multi-user system with hybrid analog/digital precoding.

to antennas structure for the AP matrix in which $\mathbf{A}$ is implemented using unit-modulus analog phase shifter, i.e, $\left|(\mathbf{A})_{i, j}\right|=1 \forall(i, j)$. Assuming coherent detection at the users, the signal-to-interference-plus-noise ratio (SINR) at user $k$ over SC $s$ is given by [4].

$$
\operatorname{SINR}_{k, s}=\frac{a_{k, s}\left|\mathbf{h}_{k, s}^{H} \mathbf{A} \mathbf{w}_{k, s}\right|^{2}}{\sum_{j \neq k} a_{j, s}\left|\mathbf{h}_{k, s}^{H} \mathbf{A} \mathbf{w}_{j, s}\right|^{2}+\sigma^{2}} .
$$

where $\sigma^{2}$ is the power of additive Gaussian noise and $h_{k, s} \in \mathbb{C}^{N_{\mathrm{T}}}$ is the frequency channel for SC $s$ from the BS to user $k, \forall(k, s)$, which can be modelled as in [3].

Considering the limitations on the system computation efforts, we are interested in jointly optimizing SA and HP design to maximize the system SR under the constraint on the maximum number of transmitted data streams. This optimization problem is stated as

$$
\begin{array}{cl}
\max _{\left\{a_{k, s}\right\},\left\{\mathbf{w}_{k, s}\right\}, \mathbf{A}} & \sum_{\forall(k, s)} \log \left(1+\operatorname{SINR}_{k, s}\right) \\
\text { s. t. } \quad & \left|(\mathbf{A})_{i, j}\right|=1, \forall(i, j) \\
& \sum_{\forall(k, s)} \mathbf{w}_{k, s}^{H} \mathbf{A}^{H} \mathbf{A} \mathbf{w}_{k, s} \leq P \\
& \sum_{\forall(k, s)} a_{k, s} \leq \bar{D},
\end{array}
$$

where $P$ is BS's transmit power budget and $\bar{D}$ is the maximum number of transmitted data streams.

\section{Two-Stage Based Solution Frameworks}

Let $p_{k, s}=\mathbf{w}_{k, s}^{H} \mathbf{w}_{k, s}$. Certainly, $p_{k, s}=0$ implies that $a_{k, s}=0$ and $p_{k, s}>0$ means that $a_{k, s}=1$. Therefore, the total number of transmitted data streams can be written mathematically as $\|\mathbf{p}\|_{0}$ where $\mathbf{p}=$ $\left[p_{1, s}, \ldots, p_{k, s}, \ldots, p_{K, S}\right]$ is the vector representing the power of all DP vectors. Thus the inequality (2d) can be transformed into a norm $\ell 0$ constraint as

$$
\|\mathbf{p}\|_{0} \leq \bar{D} \text {. }
$$

Specifically, problem (2) can be rewritten as follows.

$$
\max _{\left\{\mathbf{w}_{k, s}\right\}, \mathbf{A}} \sum_{\forall(k, s)} \log \left(1+\mathrm{SINR}_{k, s}\right) \text { s. t. (2b), (2c), (3). (4) }
$$

Based on the solution framework in [2], [3], we propose two-stage solution approach in which we aim to optimize the sparse FDP with constraint (3) being imposed, then reconstruct a near-optimal HP in the stage two.

\section{A. Stage One - Sparse FDP Optimization}

Let $\mathbf{u}_{k, s}=\mathbf{A} \mathbf{w}_{k, s}$ and $p_{k, s}^{\mathrm{F}}=\mathbf{u}_{k, s}^{H} \mathbf{u}_{k, s}$. Clearly, (3) equivalents to $\left\|\mathbf{p}^{\mathrm{F}}\right\|_{0} \leq \bar{D}$. Then, we approximate the $\ell 0$-norm of $\mathbf{p}^{\mathrm{F}}$ as $\left\|\mathbf{p}^{\mathrm{F}}\right\|_{\mathbf{0}} \approx \sum_{\forall(k, s)} f_{\mathrm{apx}}^{(k, s)}\left(p_{k, s}^{\mathrm{F}}\right)$ where $f_{\mathrm{apx}}^{(k, s)}\left(p_{k, s}^{\mathrm{F}}\right)$ is the concave function that approximates the step function of $p_{k, s}^{\mathrm{F}}$. Then, the sparse optimization problem can be expressed as

$$
\begin{aligned}
\max _{\left\{\mathbf{F}_{s}\right\}} & \sum_{\forall(k, s)} \log \left(1+\frac{\left|\mathbf{h}_{k, s}^{H} \mathbf{u}_{k, s}\right|^{2}}{\sum_{j \neq k}\left|\mathbf{h}_{k, s}^{H} \mathbf{u}_{j, s}\right|^{2}+\sigma^{2}}\right) \\
\text { s. t. } & \sum_{\forall(k, s)} \mathbf{u}_{k, s}^{H} \mathbf{u}_{k, s} \leq P \\
& \sum_{\forall(k, s)} f_{\mathrm{apx}}^{(k, s)}\left(p_{k, s}^{\mathrm{F}}\right) \leq \bar{D} .
\end{aligned}
$$

To solve this problem, we first tackle the latter obstacle by transforming the constraint $(5 \mathrm{c})$ into a linear form using the duality function [9]. Specifically, let $f_{\mathrm{cnj}}^{(k, s)}(z)$ be the conjugate function of $f_{\mathrm{apx}}^{(k, s)}(w)$, we can describe $f_{\mathrm{apx}}^{(k, s)}\left(p_{k, s}^{\mathrm{F}}\right)$ as $\hat{z}_{k, s} p_{k, s}^{\mathrm{F}}-f_{\mathrm{cnj}}^{(k, s)}\left(\hat{z}_{k, s}\right)$ where $\hat{z}_{k, s}$ is expressed as [10], [11]

$$
\hat{z}_{k, s}=\left.\nabla f_{\mathrm{apx}}^{(k, s)}(w)\right|_{w=p_{k, s}^{\mathrm{F}}} .
$$

Hence, for given $\hat{z}_{k, s}$ 's, problem (5) is approximated to

$$
\begin{aligned}
& \max _{\left\{\mathbf{u}_{k, s}\right\}} \sum_{\forall(k, s)} \log \left(1+\frac{\left|\mathbf{h}_{k, s}^{H} \mathbf{u}_{k, s}\right|^{2}}{\sum_{j \neq k}\left|\mathbf{h}_{k, s}^{H} \mathbf{u}_{j, s}\right|^{2}+\sigma^{2}}\right) \\
& \text { s. t. } \quad(5 \mathrm{~b}) \text { and } \sum_{\forall(k, s)} \hat{z}_{k, s} \mathbf{u}_{k, s}^{H} \mathbf{u}_{k, s} \leq Z
\end{aligned}
$$

where $Z=\bar{D}+\sum_{\forall(k, s)} f_{\mathrm{cnj}}^{(k, s)}\left(\hat{z}_{k, s}\right)$. Interestingly, we can address the non-convex problem (7) by relating it to a weighted sum-mean square error (MSE) minimization problem as mentioned in the following proposition.

Proposition 1. The problem (7) is equivalent to the following weighted sum-MSE minimization problem

$$
\begin{array}{cc}
\min _{\left\{\mathbf{u}_{k, s}, \delta_{k, s}, \omega_{k, s}\right\}} & \sum_{\forall(k, s)}\left(\omega_{k, s} \mathbb{E}\left[\left|x_{k, s}-\delta_{k, s} y_{k, s}\right|^{2}\right]-\log \omega_{k, s}\right) \\
\text { s.t. } \quad \text { constraints }(5 b) \text { and (7b). }
\end{array}
$$

where $\omega_{k, s}$ and $\delta_{k, s}$ denote the MSE weight and the receive coefficient for user $k$ over $S C s$, respectively. 


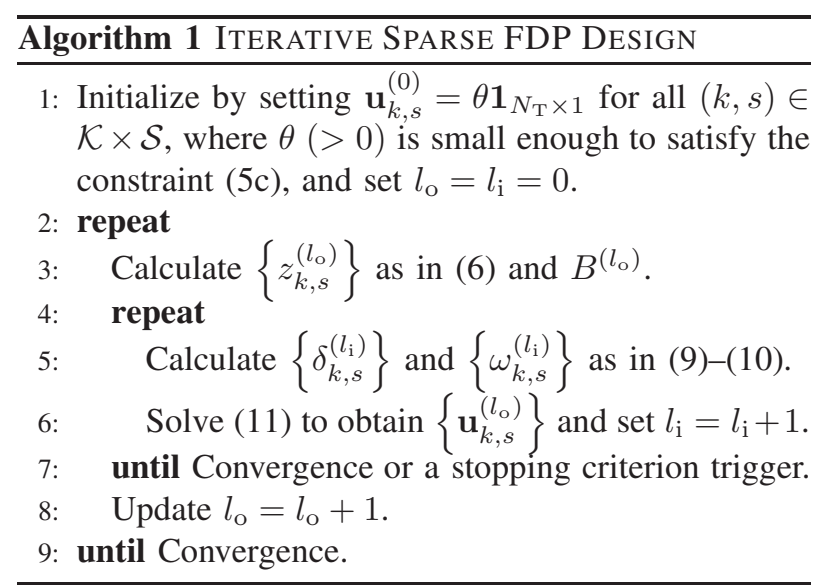

We omit the proof for brevity because it is similar to that in [12] for the case of a single sum-power constraint. It is possible to solve problem (8) by alternately optimizing over one set of variables while keeping the other two fixed.

For given FDP $\mathbf{u}_{k, s}$ 's and $\omega_{k, s}$ 's, the receive coefficient $\delta_{k, s}^{\star}$ to minimize the MSE for user $k$ over SC $s$ is the Weiner filter, i.e., MMSE receiver [12], [13]

$$
\delta_{k, s}^{\star}=\left(\sum_{j \in \mathcal{K}}\left|\mathbf{h}_{k, s}^{H} \mathbf{u}_{j, s}\right|^{2}+\sigma_{k, s}^{2}\right)^{-1} \mathbf{u}_{k, s}^{H} \mathbf{h}_{k, s} .
$$

Then, fixing $\mathbf{u}_{k, s}$ 's and $\delta_{k, s}$ 's, the MSE weights $\omega_{k, s}^{\star}$ 's can be determined as follows [12], [13].

$$
\omega_{k, s}^{\star}=e_{k, s}^{-1}=\frac{\sum_{j \in \mathcal{K}}\left|\mathbf{h}_{k, s}^{H} \mathbf{u}_{j, s}\right|^{2}+\sigma_{k, s}^{2}}{\sum_{j \in \mathcal{K} / k}\left|\mathbf{h}_{k, s}^{H} \mathbf{u}_{j, s}\right|^{2}+\sigma_{k, s}^{2}},
$$

where $e_{k, s}=\mathbb{E}\left[\left|x_{k, s}-\delta_{k, s} y_{k, s}\right|^{2}\right]$. Finally, for given $\delta_{k, s}$ 's and $\omega_{k, s}$ 's, the optimal FDP $\mathbf{u}_{k, s}$ 's can be obtained by solving the following QCQP problem.

$$
\begin{aligned}
\min _{\left\{\mathbf{u}_{k, s}\right\}} & \sum_{\forall(k, s)} \mathbf{u}_{k, s}^{H}\left(\sum_{j \in \mathcal{K}} \omega_{j, s}\left|\delta_{j, s}\right|^{2} \mathbf{h}_{j, s} \mathbf{h}_{j, s}^{H}\right) \mathbf{u}_{k, s} \\
\text { s.t. } & \text { constraints (5b), (7b). }
\end{aligned}
$$

This problem can be solved easily by employing standard optimization tools such as CVX solver [14]. By iteratively updating $\left\{\mathbf{u}_{k, s}, \delta_{k, s}, \omega_{k, s}\right\}$ 's, and $z_{k, s}$ 's, the sparse FDP can be achieved as summarized in Algorithm 1.

\section{B. Stage Two: Hybrid Precoding Design}

In this stage, we exploit an iterative algorithm to reconstruct the HP from the achieved FDP.

1) Digital Precoding Vectors Design: For a given A, the MMSE-based HP design problem can be restated as

$$
\min _{\left\{\mathbf{w}_{k, s}\right\}} \sum_{\forall(k, s)}\left\|\mathbf{u}_{k, s}^{\text {Spar }}-\mathbf{A} \mathbf{w}_{k, s}\right\|_{2}^{2} \text { s.t. (2c). }
$$

When the power constraint (2c) is temporarily removed, the well-known least squares solution can be achieved as $\hat{\mathbf{w}}_{k, s}=\mathbf{A}^{\dagger} \mathbf{u}_{k, s}^{\text {Spar }}$. Then, in order to satisfy the power constraint, the digital precoder can be normalized as

$$
\mathbf{w}_{k, s}=\sqrt{P}\left(\sum_{\forall(k, s)}\left\|\mathbf{A} \hat{\mathbf{w}}_{k, s}\right\|_{2}^{2}\right)^{-1 / 2} \hat{\mathbf{w}}_{k, s} .
$$

2) Analog Precoding Matrix Design: While fixing $\left\{\mathbf{w}_{k, s}\right\}$ 's, A can be optimized by solving

$$
\min _{\mathbf{A}} \sum_{\forall(k, s)}\left\|\mathbf{u}_{k, s}^{\mathrm{Spar}}-\mathbf{A} \mathbf{w}_{k, s}\right\|_{2}^{2} \text { s. t. }\left|(\mathbf{A})_{i, j}\right|=1 \forall(i, j) .(14)
$$

This problem is classified as a Unit-modulus Least Square type, which is non-convex and NP-hard due to the unit modulus constraints. To this end, we propose a new solution framework for this complex problem which is summarized in the Algorithm 2.

Denote $\mathbf{v}_{r}(\mathbf{A})=\operatorname{row}_{r}(\mathbf{A})^{H},\left(\mathbf{v}_{r}(\mathbf{A}) \in \mathbb{C}^{N_{\mathrm{RF}}}\right)$ where $\operatorname{row}_{r}(\mathbf{A})$ is the $r$-th row of $\mathbf{A}$. Then, the cost function of problem (14) can be rewritten as

$\Phi(\mathbf{A})=\sum_{r=1}^{N_{\mathrm{T}}}\left(\mu_{r}-2 \mathfrak{R}\left(\boldsymbol{\pi}_{r}^{H} \mathbf{v}_{r}(\mathbf{A})\right)+\mathbf{v}_{r}(\mathbf{A})^{H} \boldsymbol{\Pi}_{r} \mathbf{v}_{r}(\mathbf{A})\right)$,

where $u_{k, s, r}^{\text {Spar }}$ denotes the $r$-th element of vector $\mathbf{u}_{k, s}^{\text {Spar }}$, $\mu_{r}=\sum_{(k, s)} u_{k, s, r}^{\text {Spar }} u_{k, s, r}^{\text {Spar' }}, \pi_{r}=\sum_{(k, s)} u_{k, s, r}^{\text {Spar' }} \mathbf{w}_{k, s}$, and $\boldsymbol{\Pi}_{r}=\sum_{(k, s)} \mathbf{w}_{k, s} \mathbf{w}_{k, s}^{H}$. Problem (14) then can be decomposed into $N_{\mathrm{T}}\left(\mathcal{P}_{r}\right)$ sub-problems as

$$
\left(\mathcal{P}_{r}\right) \min _{\mathbf{v}} \mathbf{v}^{H} \boldsymbol{\Pi}_{r} \mathbf{v}-2 \mathfrak{R}\left(\boldsymbol{\pi}_{r}^{H} \mathbf{v}\right) \text { s. t. }\left|(\mathbf{v})_{i}\right|=1, \forall i .(15)
$$

This problem can be solved by the projected gradient descent-based method, where the updates of the variables are performed with the unit modulus constraint being intact. Specifically, details of this method are summarized in Algorithm 2 Steps 5-13 where the derivative of the objective function in $\left(\mathcal{P}_{r}\right)$ with respected to $\mathbf{v}_{r}$ can be calculated as $2\left(\boldsymbol{\pi}_{r}-\boldsymbol{\Pi}_{r} \mathbf{v}_{r}\right)$, and $\alpha_{r}$ 's are the step sizes. In addition, the convergence issue due to the projection onto a unit modulus constraint in Step 9 can be relieved based on the following proposition.

Proposition 2. Let $\lambda_{r}^{\max }\left(\boldsymbol{\Pi}_{r}\right)$ be the maximum eigenvalue of matrix $\Pi_{r}$. For any step size satisfying $\alpha_{r} \leq$ $\frac{1}{4 \lambda_{r}^{\max }\left(\boldsymbol{\Pi}_{r}\right)}$, the process in Step 8-12 of Algorithm 2 converges to the KKT point of the non-convex and NPhard problem $\left(\mathcal{P}_{r}\right)$.

\section{Proof: See Appendix A.}

\section{Heuristic Solution Approaches}

For comparison purposes, we consider a heuristic algorithm for solving (2). In this heuristic algorithm, the SA (i.e., $a_{k, s}$ 's) is determined first. The HP for a given SA is then designed by employing the Orthogonal Matching Pursuit method (OMP) [2].

We start with a uniform power allocation, i.e., $\mathbf{w}_{k, s}^{H} \mathbf{w}_{k, s}=p^{\text {nom }}=P / \bar{D}, \forall(k, s)$. Then, the transmission rate $R_{k, s}$ can be upper-bounded as $R_{k, s} \leq$ 


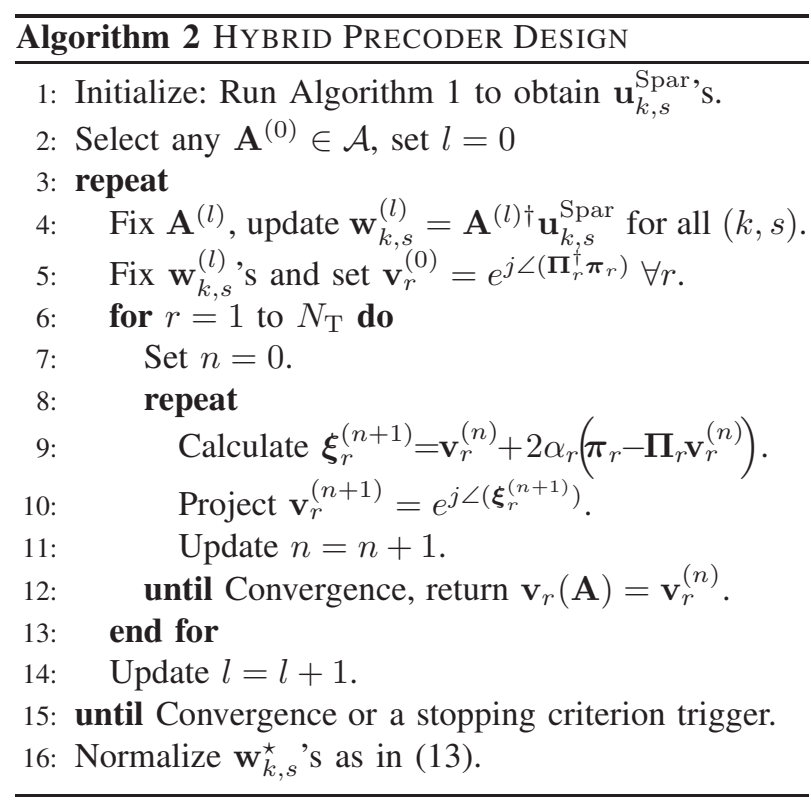

$\log \left(1+\left|\mathbf{h}_{k, s}^{H} \mathbf{A}\right|^{2} p^{\text {nom }} / \sigma^{2}\right)$. For the OPM method, columns of $\mathbf{A}$ are selected from a pre-determined set of $L_{\text {OMP }}$ basis vectors, $\mathcal{V}_{\mathrm{OMP}}=\left\{\mathbf{v}_{1}, \ldots, \mathbf{v}_{L_{\mathrm{OMP}}}\right\}$. Let $\mathbf{v}_{k, s}^{\star}=\arg \max _{\mathbf{v} \in \mathcal{V}_{\mathrm{OMP}}}\left|\mathbf{h}_{k, s}^{H} \mathbf{v}\right|^{2}$. Then, we have $R_{k, s} \leq$ $\bar{R}_{k, s}=\log \left(1+N_{\mathrm{RF}}\left|\mathbf{h}_{k, s}^{H} \mathbf{v}_{k, s}^{\star}\right|^{2} p^{\text {nom }} / \sigma^{2}\right)$. Finally, we set $a_{k, s}^{\star}$ 's corresponding to the $\bar{D}$ largest values of $\bar{R}_{k, s}$ 's to ones while keep others as zeros. After having the SA, we optimize the HP by solving the following problem.

$\max _{\left\{\mathbf{w}_{k, s}\right\}, \mathbf{A}} R_{\{a\}}^{\star}\left(\left\{\mathbf{w}_{k, s}\right\}, \mathbf{A}\right)$ s.t. constraints (2b), (2c),(16)

where $R_{\{a\}}^{\star}\left(\left\{\mathbf{w}_{k, s}\right\}, \mathbf{A}\right)=\sum_{\forall(k, s)} \log (1+$ $\left.\frac{a_{k, s}^{\star}\left|\mathbf{h}_{k, s}^{H} \mathbf{A} \mathbf{w}_{k, s}\right|^{2}}{\sum_{j \neq k} a_{j, s}^{\star}\left|\mathbf{h}_{k, s}^{H} \mathbf{A} \mathbf{w}_{j, s}\right|^{2}+\sigma^{2}}\right)$. This problem can be solved by using the OMP-HP method [2].

\section{Simulation Results}

We consider a MISO system where the BS is equipped with $8 \times 8$ UPA $(M=64)$. In this simulation, we utilize the channel vector as in [3] where the channel to each user contains of one cluster of 10 paths, all the channel path gains are assumed to be i.i.d. Gaussian distribution, and the azimuths are assumed to be uniformly distributed in $[0 ; 2 \pi]$, and the AoA/AoD elevations are uniformly distributed in $\left[-\frac{\pi}{2} ; \frac{\pi}{2}\right]$. The noise variance $\sigma^{2}$ is set at $10^{-13}$. We also employ the $5 \mathrm{G}$ mmWave path loss model for Austin $(f=38 G H z)$ as in [15] where the distances from the users to BS are set randomly between $100 \mathrm{~m}$ and $200 \mathrm{~m}$. In addition, we set $K=16, N_{R F}=16$, $\bar{D}=100, P=1.28 \mathrm{~W}$, and $S=32$.

We illustrate the convergence of our proposed algorithm in Fig. 2 where the variations of total achievable rates achieved by the FDP in stage one and the HP in stage two over the iterations are shown. As can

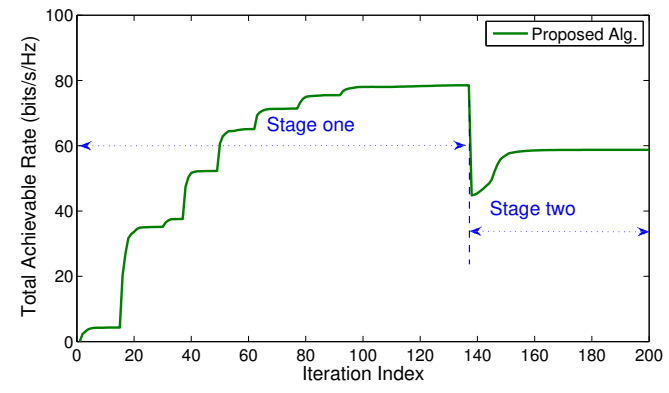

Fig. 2. Total achievable rate versus the iteration index.

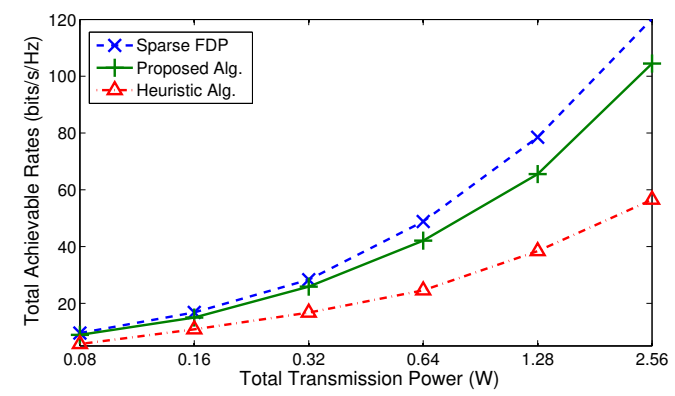

Fig. 3. Total achievable rate versus the transmission power.

be seen, the system rate in each stage increases over the iterations before reaching its the maximum value. Specifically, the convergence value of the stage one is the total achievable rates achieved by the FDP, whereas the stage two corresponds on total achievable rates achieved by the designed HP.

Fig.s 3, 4, and 5 present the total achievable rates versus the total transmission power of the base station $\left(P_{\max }\right)$, the limited number of data streams $(\bar{D})$, and the number RF chains $\left(N_{\mathrm{RF}}\right)$, respectively. For OMP designs, perfect angle of arrival (AoA) and angle of departure (AoD) codebooks are assumed. As can be seen, the achievable rates achieved by our proposed algorithm are much higher than that achieved by the heuristic one in all schemes. In addition, the SRs achieved by all algorithms increase as the transmission power, the limited number of data streams, or the number RF chains increase. Interestingly, in the high regime of $\bar{D}$, the achievable rate of all schemes will saturate as $\bar{D}$ becomes sufficiently large because of the limitation of the freedom for designing the digital precoder as illustrated in Fig. 4. Interestingly, Fig. 5 shows that our proposed algorithms can achieve the rate near to the upper bound at the high regime of RF chains which again confirms the superior performance of our proposed designs.

\section{CONCLUSION}

This paper has proposed a new joint SA and HP design for multi-user OFDMA mmWave systems under limited number of data streams. We have proposed a 


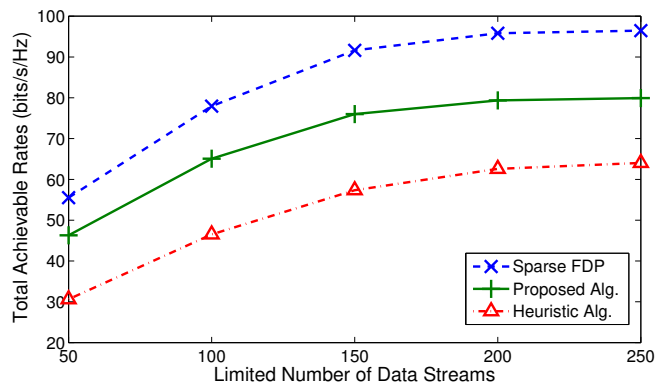

Fig. 4. Total achievable rate versus the limited number of data streams.

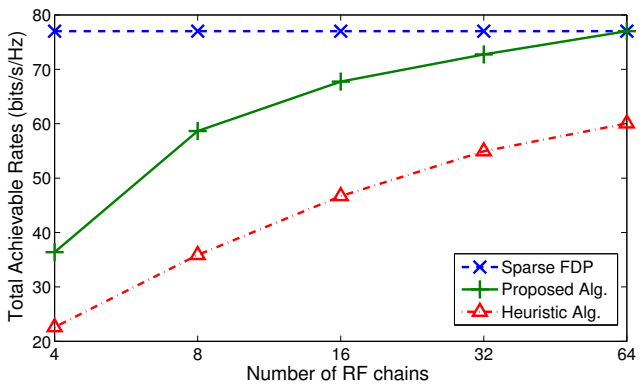

Fig. 5. Total achievable rate versus the number of RF chains.

novel two-stage solution to determined the sparse HP which aims to maximize the total achievable rate of the network. Numerical results have illustrated that our proposed algorithms outperform the reference joint SA and HP design algorithms and confirmed the efficiency of our proposed algorithms. We have also studied the impacts of various parameters on the system SR.

\section{APPENDIX A}

\section{PROOF OF PROPOSITION 2}

Recall, the gradient of $\Phi_{r}(\mathbf{v})$ can be expressed as $\nabla \Phi_{r}(\mathbf{v})=2 \boldsymbol{\Pi}_{r} \mathbf{v}-2 \boldsymbol{\pi}_{r}$, which yields

$$
\begin{aligned}
\left\|\nabla \Phi_{r}(\mathbf{v})-\nabla \Phi_{r}(\mathbf{t})\right\|_{2}^{2} & \leq 4\left\|\boldsymbol{\Pi}_{r}\right\|_{2}^{2}\|\mathbf{v}-\mathbf{t}\|_{2}^{2} \\
& \stackrel{(a)}{=} 4 \lambda_{r}^{\max }\left(\boldsymbol{\Pi}_{r}\right)\|\mathbf{v}-\mathbf{t}\|_{2}^{2} .
\end{aligned}
$$

Therefore, if we select the step size satisfying $\alpha_{r} \leq$ $1 / 4 \lambda_{r}^{\max }\left(\boldsymbol{\Pi}_{r}\right)$, we can achieve $\left\|\nabla \Phi_{r}(\mathbf{v})-\nabla \Phi_{r}(\mathbf{t})\right\|_{2}^{2} \leq$ $\frac{1}{\alpha_{r}}\|\mathbf{v}-\mathbf{t}\|_{2}^{2}$. Hence, $\nabla \Phi_{r}(\mathbf{v})$ is $\left(1 / \alpha_{r}\right)$-Lipschitz continuous. Let us introduce the new function of $\Phi_{r}(\mathbf{v})$ as

$$
\Upsilon_{r}(\mathbf{v}, \mathbf{t})=\Phi_{r}(\mathbf{t})+\nabla \Phi_{r}(\mathbf{t})^{H}(\mathbf{v}-\mathbf{t})+\frac{1}{2 \alpha_{r}}\|\mathbf{v}-\mathbf{t}\|_{2}^{2}
$$

We have $\Upsilon_{r}(\mathbf{v}, \mathbf{t}) \geq \Phi_{r}(\mathbf{v})$ and the equality holds if and only if $\mathbf{t}=\mathbf{v}$. We then can rewrite problem $\left(\mathcal{P}_{r}\right)$ as

$$
\min _{\mathbf{v}, \mathbf{t}} \Upsilon_{r}(\mathbf{v}, \mathbf{t}) \quad \text { s. t. }\left|(\mathbf{v})_{i}\right|=1 \forall i
$$

The iterative method can be employed to solve this problem where we first optimize with respect to $\mathbf{t}$ as

$$
\mathbf{t}^{(n+1)}=\arg \min _{\mathbf{t}} \Upsilon_{r}\left(\mathbf{v}^{(n)}, \mathbf{t}\right)=\mathbf{v}^{(n)} .
$$

Then, we optimize with respect to $\mathbf{v}$ as

$$
\begin{aligned}
\mathbf{v}^{(n+1)} & =\arg \min _{(\mathbf{v})_{i} \mid=1} \nabla \Phi_{r}\left(\mathbf{v}^{(n)}\right)^{H} \mathbf{v}+\frac{1}{2 \alpha_{r}}\left\|\mathbf{v}-\mathbf{v}^{(n)}\right\|_{2}^{2} \\
& =\arg \min _{\left|(\mathbf{v})_{i}\right|=1}\left\|\mathbf{v}-\mathbf{v}^{(n)}+\alpha_{r} \nabla \Phi_{r}\left(\mathbf{v}^{(n)}\right)\right\|_{2}^{2},
\end{aligned}
$$

which is exactly the same as the way we update $\mathbf{v}_{r}^{(n+1)}$ in Step 9 - 10 of Algorithm 2. In addition, we have

$$
\Upsilon_{r}\left(\mathbf{v}^{(n)}, \mathbf{t}^{(n)}\right) \geq \Upsilon_{r}\left(\mathbf{v}^{(n)}, \mathbf{t}^{(n+1)}\right) \geq \Upsilon_{r}\left(\mathbf{v}^{(n+1)}, \mathbf{t}^{(n+1)}\right) .
$$

Hence, this updating process will converge to a fixed point $\left(\mathbf{v}^{\star}, \mathbf{t}^{\star}\right)$. In addition, it is easy to see that $\Upsilon_{r}\left(\mathbf{v}, \mathbf{t}^{\star}\right) \geq \Upsilon_{r}\left(\mathbf{v}^{\star}, \mathbf{t}^{\star}\right)$ and $\Upsilon_{r}\left(\mathbf{v}^{\star}, \mathbf{t}\right) \geq \Upsilon_{r}\left(\mathbf{v}^{\star}, \mathbf{t}^{\star}\right)$, which yields that $\mathbf{v}^{\star}$ and $\mathbf{t}^{\star}$ are block-wise minimums. Hence, the process in Step 8-12 of Algorithm 2 converges to $\mathbf{v}^{\star}$ which is also a KKT point of the nonconvex and NP-hard problem $\left(\mathcal{P}_{r}\right)$.

\section{REFERENCES}

[1] J. Andrews, et al., "What will 5G be?" IEEE J. Select. Areas in Commun., vol. 32, no. 6, pp. 1065-1082, Jun. 2014.

[2] O. El Ayach, et al., "Spatially sparse precoding in millimeter wave MIMO systems," IEEE Trans. Wireless Commun., vol. 13, no. 3, pp. 1499-1513, Mar. 2014.

[3] X. Yu, et al., "Alternating minimization algorithms for hybrid precoding in millimeter wave MIMO systems," IEEE J. Sel. Topics Signal Process., vol. 10, no. 3, pp. 485-500, Apr. 2016.

[4] C. Kim, T. Kim, and J-Y. Seol, "Multi-beam transmission diversity with hybrid beamforming for MIMO-OFDM systems," in Proc. IEEE Globecom Workshops (GC Wkshps), 2013.

[5] C.-S. Sum, et al., "Virtual time-slot allocation scheme for throughput enhancement in a millimeter-wave multi-Gbps WPAN system," IEEE J. Select. Areas in Commun., vol. 27, no. 8, pp. 1379-1389, Oct. 2009.

[6] T. E. Bogale, et al., "On the number of RF chains and phase shifters, and scheduling design with hybrid analog-digital beamforming," IEEE Trans. Wireless Commun., vol. 15, no. 5, pp. 3311-3326, May 2016.

[7] E. Candes, M. Wakin, and S. Boyd, "Enhancing sparsity by reweighted $\ell_{1}$ minimization," Journal of Fourier Analysis and Applications, vol. 14, no. 5, pp. 877-905, 2008.

[8] F. Bach, et al., "Optimization with sparsity-inducing penalties," Foundations Trends Mach. Learning, vol. 4, no. 1, pp. 1-106, Jan. 2012.

[9] R. T. Rockafellar, Convex Analysis. Princeton, NJ: Princeton Univ. Press, 1970.

[10] V. N. Ha, L. B. Le, and N.-D. Dao, "Coordinated multipoint transmission design for Cloud-RANs with limited fronthaul capacity constraints" IEEE Trans. Veh. Technol., vol. 65, no. 9, pp. 7432-7447, Sep. 2016.

[11] $\mathrm{Vu} \mathrm{N}$. Ha and Long B. Le, "Joint coordinated beamforming and admission control for fronthaul constrained Cloud-RANs," in Proc. IEEE GLOBECOM'2014, Dec. 2014.

[12] S. S. Christensen, et al., "Weighted sum-rate maximization using weighted MMSE for MIMO-BC beamforming design," IEEE Trans. Wireless Commun., vol. 7, no. 12, pp. 4792-4799, Dec. 2008.

[13] V. N. Ha, D. H. N. Nguyen, and L. B. Le, "Sparse precoding design for cloud-RANs sum-rate maximization," Proc. IEEE WCNC, pp. 1648-1653, Mar. 2015.

[14] M. Grant, S. Boyd, and Y. Ye, "CVX: Matlab software for disciplined convex programming," 2009. Online: http://www.stanford.edu/ boyd/cvx.

[15] G. R. MacCartney, et al., "Path loss models for 5G millimeter wave propagation channels in urban microcells," IEEE Global Communications Conference (GLOBECOM) 2013, pp. 39483953, Dec. 2013. 